\title{
Calidad de vida relacionada con la salud, percepción de enfermedad, felicidad y emociones negativas en pacientes con diagnostico de artritis reumatoide
}

\author{
Stefano Vinaccia Alpi*1 ${ }^{*}$ Japcy Margarita Quiceno², Francy Lozano ${ }^{1}$ y Sebastian Romero ${ }^{1}$ \\ ${ }^{1}$ Fundación Universitaria SANITAS, Bogotá, Colombia. ${ }^{2}$ Universidad de Medellín, Antioquia, Colombia
}

Recibido, febrero 22/2016

Concepto de evaluación, abril 17/2016

Aceptado, julio 26/2016
Referencia: Vinaccia, S., Quiceno, J.M., Lozano, F. \& Romero, S. (2017). Calidad de vida relacionada con la salud, percepción de enfermedad, felicidad y emociones negativas en pacientes con diagnóstico de artritis reumatoide. Acta Colombiana de Psicología, 20(1), 49-59. DOI: 10.14718/ACP.2017.20.1.3

Resumen

\begin{abstract}
El propósito de este estudio fue evaluar las relaciones entre la calidad de vida relacionada con la salud (CVRS), la percepción de enfermedad, la felicidad, la ansiedad y la depresión en 62 pacientes con diagnóstico de artritis reumatoide de la ciudad de Bogotá, Colombia. El diseño del estudio fue descriptivo correlacional; se utilizaron los instrumentos Escala de calidad de vida en artritis reumatoide (QOL-RA), Cuestionario de Percepción de Enfermedad (IPQ-B), Escala Subjetiva de Felicidad (SHS) y Escala Hospitalaria de Ansiedad y Depresión (HAD); y como resultados se encontró que hubo una favorable CVRS en estos pacientes posiblemente debido a la presencia de algunas dimensiones del QOL-RA, como el apoyo, la vida social y el estado de ánimo, los cuales han demostrado tener un impacto importante sobre la calidad de vida. Con el modelo de regresión lineal múltiple se encontró un peso negativo para la ansiedad y uno positivo para la felicidad con el QOL-RA. Se concluye que estos factores psicológicos negativos y positivos tienen un peso relevante sobre la percepción de calidad de vida de los pacientes con AR.

Palabras clave: Calidad de vida relacionada con la salud, percepción de enfermedad, felicidad, ansiedad, depresión, artritis reumatoide.
\end{abstract}

\section{Health-related quality of life, illness perception, happiness and negative emotions in rbeumatoid arthritis patients}

\begin{abstract}
The aim of this study was to evaluate the relationship between quality of life related to health, illness perception, happiness, anxiety and depression in 62 patients diagnosed with rheumatoid arthritis. The study design was descriptive correlational. Instruments: The Quality of Life in Rheumatoid Arthritis Scale -QOL-RA, Brief Illness Perception Questionnaire -IPQ-B, Subjective Happiness Scale -SHS and The Hospital Anxiety and Depression Scale -HADS. Results: There was a high quality of life related to health in these patients, as some domains of QOL-RA such as support, social life and mood have demonstrated a significant impact on HRQOL. In the multiple linear regression model negative weight of anxiety and positive happiness with QOL-RA were appreciated. Conclusion: psychological factors such as anxiety and happiness have significant weight on the perceived quality of life of patients with RA.

Key words: Health-related quality of life, illness perception, happiness, anxiety, depression, rheumatoid arthritis.
\end{abstract}

Fundación Universitaria Sanitas. Carrera 7 No. 173-64. Bogotá, Colombia. vinalpi47@hotmail.com 


\title{
Qualidade de Vida Relacionada com a Saúde, Percepçáo de Doença, Felicidade e Emoçóes Negativas em Pacientes com Diagnóstico de Artrite Reumatoide
}

\begin{abstract}
Resumo
O propósito deste estudo foi avaliar as relações entre a qualidade de vida relacionada com a saúde (QVRS), a percepção de doença, a felicidade, a ansiedade e a depressão em 62 pacientes com diagnóstico de artrite reumatoide da cidade de Bogotá, Colômbia. O estudo foi descritivo correlacional; utilizaram-se os instrumentos Escala de qualidade de vida em artrite reumatoide (QDV-RA), Questionário de Percepção de Doença (IPQ-B), Escala Subjetiva de Felicidade (SHS) e Escala Hospitalar de Ansiedade e Depressão (HAD); e como resultados verificou-se que houve uma favorável CVRS nestes pacientes possivelmente devido à presença de algumas dimensões do QOL-RA, como o apoio, a vida social e o estado de ânimo, os quais demonstraram ter um impacto importante sobre a qualidade de vida. Com o modelo de regressão linear múltipla verificou-se um peso negativo para a ansiedade e um positivo para a felicidade com o QDV-RA. Conclui-se que estes fatores psicológicos negativos e positivos têm um peso relevante sobre a percepção de qualidade de vida dos pacientes com AR.

Palavras-chave: Qualidade de vida relacionada com a saúde, percepção de doença, felicidade, ansiedade, depressão, artrite reumatoide.
\end{abstract}

\section{INTRODUCCIÓN}

La definición de la artritis reumatoide (AR) se ha documentado en la literatura médica durante más de doscientos años; sin embargo, su etiología sigue siendo desconocida (Entezami, Fox, Clapmam \& Chung, 2011). A pesar de que se le han atribuido algunos posibles factores causales, como agentes infecciosos (virus, bacterias y hongos), componentes ambientales (como la adicción al tabaco) o factores genéticos, ninguno ha demostrado ser la causa de esta enfermedad (Julia \& Marsal, 2013; Pratt \& Isaacs, 2015).

La AR afecta aproximadamente al 1\% de la población mundial, y a medida que avanza la enfermedad, las consecuencias para la salud del paciente se identifican por la pérdida de la movilidad articular, deformidad, discapacidad, dolor crónico y la presencia de emociones negativas como la ansiedad, la depresión, el estrés y la desesperanza (Gibofsky, 2012; Santiago, Geenen, Jacobs \& Da Silva, 2015; Zhao, Chen \& Chen, 2015), además de presentar dependencia física y emocional, fatiga, rigidez matutina y trastornos del sueño, entre otros (Purabdollah, Lakdizaji, Rahmani, Hajaliu \& Anzarin, 2015).

En Colombia, los estudios epidemiológicos han estimado una prevalencia de la AR entre 0.8-1.0\%; con una edad promedio de aparición de 53.2 años y de inicio de los síntomas de 41.3; una mayor prevalencia en el sexo femenino (81.9\%); y una comorbilidad relacionada con la hipertensión esencial, la osteoporosis y el síndrome de Sjögren (Bautista et al., 2015; Machado, Ruiz \& Medina, 2015).

Por estas razones, la AR implica un cambio importante en la calidad de vida relacionada con la salud (CVRS) de las personas que la padecen (Matcham, Scott, Rayner \&
Hotopf, 2014). La CVRS es entendida como la valoración que realiza una persona, de acuerdo con sus propios criterios, de su estado físico, emocional y social en un momento dado, y el reflejo del grado de satisfacción con una situación personal a nivel fisiológico (sintomatología general, discapacidad funcional, situación analítica, sueño o respuesta sexual), emocional (sentimientos de tristeza, miedo, inseguridad o frustración), y social (situación laboral o escolar, interacciones sociales en general, relaciones familiares, amistades, nivel económico, participación en la comunidad o actividades de ocio, entre otras) (Schwartzmann, 2003).

La persona con diagnóstico de AR debe enfrentar no solo los síntomas asociados a la enfermedad, sino también la ambigüedad de los mismos (Purabdollah et al., 2015), dado que esta enfermedad es una de las principales condiciones en las que más se disminuye la calidad de vida (Garip, Eser \& Bodur, 2011). Sin embargo, el predominio de los síntomas ante situaciones de deterioro funcional y físico no excluye la presencia de emociones positivas (como la felicidad), las cuales sirven como marcos protectores para la salud física y mental de pacientes con condiciones médicas crónicas (Angner, Ray, Saag \& Allinson, 2009; Angner, Ghandhi et al., 2013; Garaigordobil, 2015).

Según Palomera (2009), “la felicidad está constituida por tres dimensiones: emociones positivas, emociones negativas y satisfacción vital [...] y es el resultado del grado en que las experiencias de afectividad positiva superan las experiencias de afectividad negativa y del grado en el que una persona percibe que sus metas y motivaciones están resueltas.” (p. 273). Esta capacidad de mantener la emocionalidad positiva durante los períodos de enfermedad se ha asociado con un mejor bienestar (Lyubomirsky, Sheldon \& Schkade, 2005; 
Steptoe \& Wardle, 2005), y la literatura es consistente en afirmar las relaciones positivas entre la calidad de vida y la felicidad en la salud (Musschenga, 1997).

Además, los factores cognitivos y emocionales juegan un papel relevante en la percepción del paciente sobre su enfermedad, lo cual influye en la evolución y curso de la misma. Concretamente, la percepción de la enfermedad se refiere a la representación cognitiva y emocional que el paciente hace de su condición, en donde si esta implica una amenaza para su salud, la representación orienta las acciones y el uso de estrategias de afrontamiento para sobrellevarla (Quiceno \& Vinaccia, 2010).

En este sentido, la percepción de la enfermedad está relacionada con resultados en la AR que incluyen la discapacidad, el bajo estado de ánimo, el dolor y el deterioro en la función física (Prajapati et al., 2014); y puede retrasar la búsqueda del tratamiento médico, lo que puede agudizar los síntomas de la enfermedad y conducir en el largo plazo a la pérdida de funciones de la vida diaria, como la capacidad de desplazamiento, el cuidado personal, la capacidad para el trabajo, la independencia económica y la participación en los roles sociales, entre otras actividades básicas (Van der Elst et al., 2015). En diferentes estudios se ha encontrado una relación directamente proporcional entre la CVRS y la percepción de la enfermedad (Quiceno \& Vinaccia, 2010).

De acuerdo con lo anterior, el objetivo de este estudio fue evaluar las relaciones entre la calidad de vida relacionada con la salud, la percepción de enfermedad, la felicidad, la ansiedad y la depresión en 62 pacientes con diagnóstico de artritis reumatoide residentes en la ciudad de Bogotá.

\section{MÉTODO}

\section{Participantes}

En este estudio participaron 62 pacientes adultos (hombres y mujeres) con diagnóstico de artritis reumatoide según los criterios de clasificación del año 2010. La muestra fue seleccionada mediante un muestreo no aleatorio de los sujetos disponibles pertenecientes a una fundación de la ciudad de Bogotá que presta apoyo al paciente reumático. Como criterios de inclusión se tuvo en cuenta que fueran mayores de 18 años, que no presentaran diagnóstico de alguna limitación cognitiva y que aceptaran de forma libre y voluntaria su participación en el estudio.

\section{Instrumentos}

Escala de calidad de vida en artritis reumatoide (Quality of Life in Rheumatoid Arthritis Scale, QOL-RA). El QOLRA es un cuestionario específico que evalúa la CVRS de pacientes con artritis reumatoide desarrollado originalmente y validado al español por Danao, Padilla y Johnson (2001) cuyos coeficientes alfa de Cronbach van de .87 a.90. En esta investigación se utilizó la versión colombiana de Vinaccia, Riveros, Quiceno y Anaya (en prensa) que contiene 8 ítems, en donde cada uno conforma una dimensión, por lo cual no se efectúa el análisis alfa de Cronbach. Sus ocho dimensiones son: habilidad física, apoyo, dolor, tensión nerviosa, salud, artritis, vida social y estado de ánimo; además, contiene una escala total. El sistema de respuesta es tipo Likert que va de 0 (muy mala calidad de vida) a 10 puntos (excelente calidad de vida) en donde a mayor puntuación, mejor percepción de CVRS. El coeficiente alfa de Cronbach de la escala total en este estudio fue de .949.

Cuestionario de Percepción de Enfermedad (The Brief Illness Perception Questionnaire, IPQ-B). Desarrollado originalmente por Broadbent, Petrie, Main y Weinman (2006). Contiene 8 ítems en donde cada uno conforma una dimensión, y por tanto no se efectúa el análisis alfa de Cronbach. El sistema de respuesta es tipo Likert que va de 0 a 10 puntos y cuenta con las dimensiones: consecuencias, duración, control personal, control del tratamiento, identidad, preocupación, respuesta emocional y comprensión de la enfermedad.

Escala Subjetiva de Felicidad (Subjective Happiness Scale, SHS). Elaborada originalmente por Lyubomirsky y Lepper (1999). Cuenta con un nivel alto de fiabilidad ( $\alpha$ $<.80$ ), comprende 4 ítems que se responden en un sistema tipo Likert que va de 1 a 7 puntos, en donde a mayor puntuación, mayor percepción subjetiva de felicidad. El coeficiente alfa de Cronbach para este estudio fue de .711.

Escala Hospitalaria de Ansiedad y Depresión (The Hospital Anxiety and Depression Scale, HADS). La versión colombiana de la escala HADS fue desarrollada por Rico, Restrepo y Molina (2005), cuenta con un coeficiente alfa de Cronbach de .85, se compone por 14 ítems que se responden en un sistema tipo Likert con 4 opciones de respuesta que van de 0 a 3 puntos, y cuenta con dos dimensiones que evalúan la ansiedad y la depresión con relación al periodo actual. El rango para cada dimensión es de 0 a 21 puntos, en donde a mayor puntuación, más altos niveles de ansiedad o depresión. El coeficiente alfa de Cronbach para este estudio fue de .824 para ansiedad y de .795 para depresión.

\section{Procedimiento}

Una vez se contó con el permiso y la aceptación de las directivas y el comité científico de la fundación que apoyó la investigación, se convocó a los pacientes a una reunión para invitarlos a participar en el estudio. Los que acogieron la invitación fueron reunidos en las instalaciones de la fundación en grupos de máximo veinte personas. A 
cada grupo se les aclararon los propósitos y alcances de la investigación para que luego, de manera libre y voluntaria, firmaran el consentimiento informado. Posteriormente, de forma auto administrada y contando con la ayuda de los investigadores a cargo del estudio cuando los pacientes lo requirieron, cada paciente respondió los cuestionarios (QOL-RA, IPQ-B, SHS y HADS) y una ficha de datos sociodemográficos. El tiempo de aplicación fue de, en promedio, una hora para cada participante.

\section{Análisis de resultados}

Los resultados del estudio fueron analizados a través del paquete estadístico SPSS versión 22. Para el análisis descriptivo de las variables edad, tiempo de diagnóstico, felicidad, ansiedad, depresión, percepción de enfermedad y CVRS se utilizó como medida de tendencia central la media. Así mismo, se calculó la desviación típica (DT) y las puntuaciones mínimas (Mín.) y máximas (Máx.) de las diferentes dimensiones de los instrumentos psicométricos utilizados. Con respecto a las variables nominales (sexo, estado civil, nivel educativo, estrato socioeconómico, ocupación, con quien vive, sistema de salud, toma medicamentos psiquiátricos y específicos para la enfermedad) se emplearon porcentajes.

Además, se calculó el coeficiente alfa de Cronbach para el análisis de fiabilidad de los instrumentos psicométricos del estudio, el coeficiente $r$ de Pearson para el análisis correlacional entre edad y tiempo de diagnóstico (variables sociodemográficas) y las diferentes dimensiones de los cuestionarios de calidad de vida relacionada con la salud (QOL-RA, IPQ-B, SHS y HADS), calculándose previamente la distribución de las variables (Kolmogorov-Smirnov). Por último, se empleó un modelo de regresión lineal múltiple considerando el método de selección por pasos para analizar el peso de las variables predictoras (edad, tiempo de diagnóstico, felicidad, ansiedad, depresión, y percepción de enfermedad) sobre la variable criterio (CVRS).

\section{RESULTADOS}

\section{Análisis descriptivo según las características sociodemo- gráficas}

En la Tabla 1 se presentan los datos descriptivos de las variables sociodemográficas y clínicas de la investigación. Para este estudio se encuentra un predominio del sexo femenino respecto al masculino, con una edad media de $\mathrm{M}=59.7$ años, $\mathrm{y}$ un rango entre los 32 a 79 . Con respecto al estado civil, hubo predominio de la muestra en las categorías casado (30.6\%) y separado/divorciado (30.6\%); la mayoría alcanzó un nivel educativo de básica secundaria (bachillerato) (41.9\%) y pertenece a un estrato socioeconómico colombiano medio bajo (nivel 1, 2 y 3; 75.8\%). De igual modo gran parte de la muestra se encuentra retirada de su vida laboral (48.4\%) y un porcentaje menor se dedica a actividades del hogar (30.6\%). Además, gran proporción de los participantes viven con familiares (90.3\%), y son muy pocos los que viven solos (9.7\%).

Con respecto a las variables clínicas, se observa que los pacientes tienen un tiempo promedio de diagnóstico de AR de 17 años, en un rango que oscila entre los 2 y los 60 años de diagnóstico. La mayoría (77.4\%) cuenta con un sistema de salud de EPS (Empresa Promotora de Salud) y no consumen medicación psiquiátrica (79\%), aunque sí cuentan con un tratamiento medicamentoso específico para su enfermedad (85.5\%).

Análisis descriptivo según las variables psicológicas del estudio

En la Tabla 2 se presentan los datos descriptivos obtenidos de los instrumentos empleados.

A nivel general se encontró una fiabilidad entre aceptable y muy buena en las diferentes dimensiones y escalas totales de los instrumentos psicométricos utilizados en el estudio, tal como se aprecia en la Tabla 2.

Con respecto a los resultados descriptivos, se observa que la felicidad (SHS), tuvo una puntuación promedio. En cuanto a la escala HADS, según los criterios de Rico et al. (2005) para indicar si los pacientes en Colombia presentan o no la sintomatología, se encontró que en la subescala de ansiedad hubo una puntuación que limitó con el punto de corte (8), mientras que en la subescala de depresión se encontró una puntuación baja con respecto al punto de corte (9). En la percepción de enfermedad (IPQ-B), las puntuaciones medias más altas se encontraron en las dimensiones duración, control de tratamiento y comprensión de la enfermedad, mientras que las dimensiones consecuencias, control personal, identidad, preocupación y respuesta emocional tuvieron una puntuación moderada. Y en cuanto a la escala QOL-RA de CVRS se observan las puntuaciones medias más altas en las dimensiones apoyo, vida social, y estado de ánimo; y puntuaciones promedio en las dimensiones habilidad física, dolor, tensión nerviosa, salud y artritis, además de la escala total QOL-RA.

\section{Análisis de correlación de Pearson}

En la Tabla 3 se presentan los resultados del análisis de correlación de Pearson entre la edad, tiempo de diagnóstico, felicidad (HSH), ansiedad (HAD), depresión (HAD), y percepción de enfermedad (IPQ-B) con la CVRS (QOL-RA), 
Tabla 1

Características sociodemográficas y clínicas de la muestra $(n=62)$

Factores Sociodemográficos

\section{n $\%$}

Sexo

\begin{tabular}{lcl}
\multicolumn{2}{c}{ Sexo } & \\
\cline { 1 - 2 } & 3 & 4.8 \\
Mombre & 59 & 95.2
\end{tabular}

Estado civil

\begin{tabular}{l}
\hline Soltero \\
Casado \\
Separado/Divorciado \\
Unión libre \\
Viudo $\quad$ Estrato socioeconómico \\
\hline 1 \\
2 \\
3 \\
4 \\
5 \\
6
\end{tabular}

\begin{tabular}{lll}
\hline Solo & 6 & 9.68 \\
Con familia & 56 & 90.32
\end{tabular}

$12 \quad 19.4$

$19 \quad 30.6$

$19 \quad 30.6$

$5 \quad 8.1$

$\begin{array}{ll}7 & 11.3\end{array}$

23.2

$29 \quad 46.8$

$13 \quad 21.0$

$1 \quad 1.6$

$1 \quad 1.6$
$16 \quad 25.8$ n $\quad \%$

Edad

\begin{tabular}{lll}
\hline Media (DT) & 59.7 & 9.3 \\
Mín.-Máx. & 32 & 79
\end{tabular}

Nivel educativo

Primaria 15

Secundaria

$26 \quad 41.9$

Tecnología

$11 \quad 17.7$

Universitario

$7 \quad 11.3$

Posgrado

3

4.8

Ocupación

Empleado $\quad 4 \quad 6.5$

$\begin{array}{lll}\text { Trabajador independiente } & 7 & 11.3\end{array}$

Jubilado/pensionado $\quad 30 \quad 48.4$

Desempleado $2 \quad 3.2$

Hogar

$19 \quad 30.6$

\begin{tabular}{|c|c|c|c|c|c|}
\hline \multicolumn{6}{|c|}{ Factores clínicos } \\
\hline & $\mathrm{n}$ & $\%$ & & $\mathrm{n}$ & $\%$ \\
\hline Sistema de Salud & & & Tiempo de Diagnóstico & & \\
\hline SISBEN & 5 & 8.1 & Media (D.T) & 205.7 & 170.3 \\
\hline EPS & 48 & 77.4 & Mín.-Máx. & 23 & 720 \\
\hline Medicina prepagada & 8 & 12.9 & & & \\
\hline Particular/Privada & 1 & 1.6 & & & \\
\hline Toma medicamentos psiquiátricos & & & $\begin{array}{l}\text { Recibe tratamiento especifico para } \\
\text { la enfermedad }\end{array}$ & & \\
\hline SI & 13 & 21.0 & SI & 53 & 85.5 \\
\hline NO & 49 & 79.0 & NO & 9 & 14.5 \\
\hline
\end{tabular}

Nota. n= número de participantes; DT= Desviación Típica; Mín.= Mínimo; Máx.= Máximo. 
Tabla 2

Estadísticos descriptivos de las variables felicidad, ansiedad, depresión, percepción de enfermedad y CVRS

\begin{tabular}{|c|c|c|c|c|}
\hline & Media & DT & Mín. & Máx. \\
\hline SHS: Felicidad & 4.06 & 1.02 & 2 & 6 \\
\hline HAD: Ansiedad & 8.34 & 4.11 & 0 & 18 \\
\hline HAD: Depresión & 5.69 & 3.60 & 0 & 14 \\
\hline \multicolumn{5}{|l|}{$\begin{array}{l}\text { IPQ-B: Percepción de } \\
\text { enfermedad }\end{array}$} \\
\hline Consecuencias & 6.08 & 2.52 & 0 & 10 \\
\hline Duración & 8.31 & 2.49 & 0 & 10 \\
\hline Control personal & 6.63 & 2.63 & 0 & 10 \\
\hline Control tratamiento & 7.65 & 2.33 & 0 & 10 \\
\hline Identidad & 6.06 & 2.37 & 0 & 10 \\
\hline Preocupación & 6.34 & 2.69 & 0 & 10 \\
\hline $\begin{array}{l}\text { Comprensión de la } \\
\text { enfermedad }\end{array}$ & 7.98 & 1.87 & 0 & 10 \\
\hline Respuesta emocional & 6.45 & 2.86 & 0 & 10 \\
\hline \multicolumn{5}{|l|}{ QOL-RA: CVRS en Artritis } \\
\hline Habilidad física & 6.40 & 2.38 & 1 & 10 \\
\hline Ароуо & 7.39 & 2.25 & 1 & 10 \\
\hline Dolor & 5.89 & 2.61 & 1 & 10 \\
\hline Tensión nerviosa & 6.77 & 2.66 & 1 & 10 \\
\hline Salud & 6.16 & 2.34 & 1 & 10 \\
\hline Artritis & 6.06 & 2.60 & 1 & 10 \\
\hline Vida social & 7.13 & 2.53 & 1 & 10 \\
\hline Estado de ánimo & 7.05 & 2.43 & 1 & 10 \\
\hline Escala total QOL-RA & 6.62 & 2.13 & 1 & 10 \\
\hline
\end{tabular}

Nota. DT= desviación típica; Mín.= mínimo; Máx.= máximo.

en donde se encontró relación estadísticamente significativa al nivel de .01 y .05 entre algunas de las variables.

Con relación a la Escala de Felicidad (SHS) se observan correlaciones positivas moderadas y altas con todas las dimensiones del cuestionario de CVRS (QOL-RA), como con habilidad física, apoyo, dolor, tensión nerviosa, salud, artritis, vida social y estado de ánimo además de la escala total. De igual modo, la escala HAD de ansiedad y depresión tuvo correlaciones negativas moderadas y altas con todas las dimensiones de CVRS.

Con respecto al Cuestionario de Percepción de Enfermedad(IPQ-B) se observa que las dimensiones control personal, identidad y preocupación tuvieron correlaciones negativas bajas y moderadas con todas las dimensiones de CVRS. Por otro lado, la dimensión consecuencias tuvo correlaciones negativas bajas y moderadas con todas las dimensiones de CVRS, menos con la dimensión tensión nerviosa; de igual modo, la dimensión respuesta emocional tuvo correlaciones negativas bajas y moderadas con todas las dimensiones de CVRS, menos con la dimensión dolor. Por último, la dimensión control de tratamiento del IPQ-B tuvo una correlación negativa baja con la dimensión estado de ánimo de CVRS.

\section{Análisis de regresión lineal múltiple}

De acuerdo con los resultados encontrados en los modelos finales de regresión lineal múltiple, en donde se consideró como variable dependiente (VD) la CVRS (QOL-RA), esta presentó un coeficiente de determinación $R^{2} c=49.4 \%$, explicado negativamente por la dimensión ansiedad de la escala HAD $(\beta=-0.453 ; t=-4.241 ; p=.000)$ y positivamente por la Escala de Felicidad (SHS) $(\beta=0.364 ; t=3.411$; $p=.001)$ (Tabla 4).

\section{DISCUSIÓN}

En este estudio participaron, en su mayoría, mujeres con una edad media cercana a los 60 años, con estudios de educación secundaria, de estado civil entre casada o divorciada/separado, jubiladas, pertenecientes al estrato socioeconómico colombiano medio/bajo y, en promedio, con 16 años y 6 meses de diagnóstico de artritis reumatoide.

Cabe resaltar que algunos de los instrumentos utilizados en este estudio no han sido validados en el contexto colombiano; se cuenta solo con caracterizaciones de los cuestionarios IPQ-B de percepción de enfermedad (Vinaccia, Quiceno \& Remor, 2012) y la escala subjetiva de felicidad (SHS) (Quiceno et al., 2012; 2013), mientras que, por otro lado, se cuenta con la validación colombiana de la escala hospitalaria de ansiedad y depresión (HADS) (Rico et al., 2005) y la escala de calidad de vida en artritis reumatoide (QOL- RA) (Vinaccia, et al., en prensa).

En cuanto a los resultados descriptivos del estudio, de acuerdo con la percepción de enfermedad (IPQ-B), los pacientes saben que su enfermedad tiene un pronóstico de larga duración y comprenden en qué consiste, reportan la presencia de síntomas y signos de su afección que los limitan de cierta manera en su vida diaria, perciben que tienen un control moderado sobre el tratamiento médico, e indican que en la medida de sus capacidades tratan de manejar su enfermedad. Por otro lado, también perciben que la enfermedad les afecta en algo su vida y presentan 
Tabla 3

Análisis de correlación de Pearson entre edad, tiempo de diagnóstico, felicidad, ansiedad y depresión, percepción de enfermedad con CVRS

\begin{tabular}{|c|c|c|c|c|c|c|c|c|c|}
\hline \multicolumn{10}{|c|}{ QOL-RA } \\
\hline & $\begin{array}{l}\text { Habilidad } \\
\text { física }\end{array}$ & Ароуо & Dolor & $\begin{array}{l}\text { Tensión } \\
\text { nerviosa }\end{array}$ & Salud & Artritis & $\begin{array}{l}\text { Vida } \\
\text { social }\end{array}$ & $\begin{array}{l}\text { Estado de } \\
\text { ánimo }\end{array}$ & $\begin{array}{l}\text { Escala Total } \\
\text { QOL-RA }\end{array}$ \\
\hline Edad & -.054 & .112 & .061 & -.067 & -.018 & .044 & .155 & -.116 & .016 \\
\hline Tiempo de Diagnóstico & .009 & .185 & .1 & .235 & -.015 & .094 & .044 & .078 & .106 \\
\hline SHS: Felicidad & $.433^{* *}$ & $.515^{* *}$ & $.420^{* *}$ & $.563^{* *}$ & $.505^{* *}$ & $.439^{* *}$ & $.623^{* *}$ & $.640^{* *}$ & $.601^{* *}$ \\
\hline HAD Ansiedad & $-.492^{* *}$ & $-.454^{* *}$ & $-.489^{* *}$ & $-.581^{* *}$ & $-.624^{* *}$ & $-.510^{* *}$ & $-.593^{* *}$ & $-.674^{* *}$ & $-.644^{* *}$ \\
\hline HAD Depresión & $-.489^{* *}$ & $-.493^{* *}$ & $-.410^{* *}$ & $-.549^{* *}$ & $-.509^{* *}$ & $-.475^{* *}$ & $-.481^{* *}$ & $-.666^{* *}$ & $-.591^{* *}$ \\
\hline IPQ-B: Consecuencias & $-.467^{* *}$ & $-.442^{* *}$ & $-.262^{*}$ & -.234 & $-.432^{* *}$ & $-.298^{*}$ & $-.440^{* *}$ & $-.369^{* *}$ & $-.424^{* *}$ \\
\hline IPQ-B: Duración & -.071 & .04 & .116 & .124 & -.037 & .098 & .001 & .016 & .044 \\
\hline $\begin{array}{l}\text { IPQ-B: Control } \\
\text { personal }\end{array}$ & $.437^{* *}$ & $.355^{* *}$ & $.422^{* *}$ & $.314^{*}$ & $.431^{* *}$ & $.325^{* *}$ & $.353^{* *}$ & $.545^{* *}$ & $.458^{* *}$ \\
\hline $\begin{array}{l}\text { IPQ-B: Control } \\
\text { tratamiento }\end{array}$ & .171 & .102 & .184 & .106 & .227 & .16 & .191 & $.295^{*}$ & .209 \\
\hline IPQ-B: Identidad & $-.530^{* *}$ & $-.431^{* *}$ & $-.366^{* *}$ & $-.267^{*}$ & $-.477^{* *}$ & $-.398^{* *}$ & $-.419^{* *}$ & $-.364^{* *}$ & $-.470^{* *}$ \\
\hline IPQ-B: Preocupación & $-.312^{*}$ & $-.334^{* *}$ & $-.312^{*}$ & $-.395^{* *}$ & $-.403^{* *}$ & $-.345^{* *}$ & $-.375^{* *}$ & $-.417^{* *}$ & $-.424^{* *}$ \\
\hline $\begin{array}{l}\text { IPQ-B: Comprensión } \\
\text { de la Enfermedad }\end{array}$ & -.002 & .107 & .11 & .003 & .072 & -.01 & .111 & .141 & .073 \\
\hline $\begin{array}{l}\text { IPQ-B: Respuesta } \\
\text { Emocional }\end{array}$ & $-.474^{* *}$ & $-.403^{* *}$ & -.215 & $-.353^{* *}$ & $-.421^{* *}$ & $-.328^{* *}$ & $-.480^{* *}$ & $-.468^{* *}$ & $-.455^{* *}$ \\
\hline
\end{tabular}

Nota. ${ }^{*} p<.05$, dos colas; ${ }^{* *} p<.01$, dos colas.

cierta preocupación por las consecuencias que les pueda acarrear a nivel físico, social, económico y emocional; y manifiestan respuestas emocionales como rabia, enojo, susto y tristeza.

Con respecto a la felicidad (SHS), los participantes de este estudio tuvieron puntuaciones más bajas $(M=4.06)$ en comparación a otras investigaciones desarrolladas con la misma escala en Colombia con muestras de estudiantes universitarios ( $M=5.0$ ) (Quiceno et al., 2013) y con hombres privados de la libertad ( $M=5.0$ ) (Quiceno et al., 2012). Al respecto, en los pacientes con AR de este estudio los niveles de felicidad no son tan favorables en comparación a los de muestras sanas colombianas a pesar de que diferentes investigaciones plantean que la población colombiana tiene índices muy altos de felicidad y optimismo, independientemente de los acontecimientos negativos o positivos que les acontezcan (CENEC, 2001; Cruz \& Torres, 2006; Sinisterra, 2007).

Por otro lado, con respecto a los niveles de ansiedad y depresión (HADS) a nivel general no se encontraron síntomas clínicos de depresión en los pacientes (Rico et al., 2005), aunque sí los hubo de ansiedad, pues las puntuaciones estuvieron ligeramente por encima del punto de corte para muestras colombianas $(M=8.34)$. Estos resultados difieren en relación con los síntomas de depresión hallados en pacientes con AR del estudio de Matcham et

Tabla 4

Análisis regresión lineal múltiple

\begin{tabular}{llllll}
\hline & \multicolumn{2}{l}{ Coeficientes no estandarizados } & \multicolumn{3}{l}{ Coeficientes estandarizados } \\
\hline & $B$ & Error estándar & Beta & $t$ & Sig. \\
(Constante) & 5.507 & 1.224 & & 4.498 & .000 \\
Ansiedad -HAD & -.235 & .055 & -.453 & -4.241 & .000 \\
Felicidad -SHS & .758 & .222 & .364 & 3.411 & .001 \\
\hline
\end{tabular}


al. (2014), pero se asemejan a los resultados del estudio de Ryan (2014) con respecto a los niveles de ansiedad hallados en pacientes con RA.

Con respecto a la CVRS (QOL-RA), los pacientes de este estudio tuvieron puntuaciones medias más altas en las dimensiones apoyo, vida social y estado de ánimo, y puntuaciones promedio en las dimensiones habilidad física, dolor, tensión nerviosa, salud, artritis, y en la escala total QOL-RA. Los resultados de la escala total del QOL-RA en este estudio de pacientes nativos de la ciudad de Bogotá $(M=6.62)$ son ligeramente similares a los alcanzados en los estudios de Vinaccia, Fernández, Moreno y Padilla (2006) $(M=6.40)$ y difieren un poco al estudio de Vinaccia, Tobón, Moreno-San Pedro, Cadena y Anaya (2005) $(M=5.28)$ con muestras de AR nativos de la cultura paisa de la ciudad de Medellín. A pesar de las diferencias en el estadio de la enfermedad y la cultura en estos tres estudios, se aprecia que los niveles de CVRS se conservan casi similares, es decir, las diferencias culturales en este tipo de enfermedad no son tan marcadas en muestras colombianas, lo que difiere de otros estudios que han planteado la influencia de los factores étnicos y culturales en la percepción de la calidad de vida tanto en población general como con alguna enfermedad crónica (Goh, Rusli \& Khalid, 2014; Verhagen, Ros, Steunenberg \& De Witt, 2014).

En cuanto al análisis de correlación se aprecia que para estos pacientes no se encontraron correlaciones significativas entre edad y tiempo de diagnóstico de la enfermedad con la CVRS. En cuanto a la escala de percepción de enfermedad (IPQ-B), las dimensiones duración, control de tratamiento y comprensión de la enfermedad no tuvieron correlaciones con la CVRS, sin embargo, las demás dimensiones consecuencias, identidad, preocupación y respuesta emocional se asociaron negativamente con las diferentes dimensiones y escala total de la CVRS (QOL-RA), y la dimensión control personal tuvo una correlación positiva con la CVRS. Al respecto, Quiceno y Vinaccia (2010) encontraron en una revisión de literatura con diferentes patologías físicas que la percepción de enfermedad se correlacionaba con la calidad de vida, evidenciando así que las percepciones negativas de la enfermedad tienen un impacto directo sobre el bienestar físico y mental de personas con alguna patología crónica enfermos crónicos (Fowler \& Baas, 2006; Groarke, Curtis, Coughlan \& Gsel, 2004; Paschalides et al., 2004).

De igual modo, se encuentra que la escala HADS, que mide ansiedad y depresión, tuvo correlaciones negativas con todas las dimensiones y escala total del QOL-RA. Lo cual se relaciona con diferentes estudios que han puesto de manifiesto niveles significativos $(21 \%$ a $70 \%)$ de la ansiedad en la AR (Covic et al., 2012; Isik, Koca, Ozturk
\& Mermi, 2007) y la influencia de esta emoción negativa sobre la CVRS (Nas et al., 2011; Ozcetin et al., 2007).

Con relación a la escala de felicidad (SHS) se observan correlaciones positivas con todas las dimensiones y la escala total del QOL-RA. Al respecto, la investigación del constructo felicidad como bienestar subjetivo se ha desarrollado en la psicología de la salud como un importante indicador de calidad de vida en muestras de pacientes crónicos en proceso de hemodiálisis (Bennet, Weinberg, Bridgman \& Cummins, 2015; Musschenga, 1997), en personas de la tercera edad con diferentes diagnósticos médicos (Steptoe, Deaton \& Stone, 2015) y en personas con esquizofrenia (Palmer, Martin, Deeep, Glorioso \& Jeste, 2014).

Por otro lado, en el análisis de regresión lineal múltiple se aprecia que la dimensión ansiedad de la escala HADS tuvo un peso negativo sobre la calidad de vida, mientras que la felicidad de la escala SHS tuvo un peso positivo sobre la misma. Resultados similares se han encontrado en muestras de enfermos crónicos (Hoppe, 2013; Nas et al., 2011; Ozcetin et al., 2007; Veenhoven, 2008) y en pacientes con dolor crónico (Muller et al., 2015; Tran et al., 2015; Ryan \& McGuire, 2015).

Como limitaciones del estudio se encuentran, en primer lugar, el tamaño limitado de la muestra (62 personas), que estuvo condicionado a las características patológicas (diagnóstico de artritis reumatoide) de los participantes del estudio; en segundo lugar, la ausencia de estudios epidemiológicos sólidos sobre esta enfermedad en Colombia y sobre la población con AR de la ciudad de Bogotá; y, en tercer lugar, la falta de instrumentos validados para la población de estudio.

Finalmente, los resultados de esta investigación evidencian la importancia del estudio de variables salutogénicas (felicidad) y patogénicas (ansiedad y depresión) sobre la CVRS de las personas con AR. El diagnóstico de una enfermedad crónica como esta afecta profundamente la vida de las personas que las padecen, pues su proyecto de vida deberá ser revisado y a menudo no podrán saber con certeza el curso de la enfermedad, dado que sus características de evolución son, en muchos casos, impredecibles (Vinaccia, Ramírez \& Toro, 2001). Por otro lado, se han encontrado en estos pacientes algunos déficits en la desesperanza aprendida (tanto a nivel motivacional, por la reducción de las actividades de la vida diaria; como a nivel cognitivo, por la reducción de esfuerzos para desarrollar nuevas estrategias de adaptación), que llegan a afectar el área emocional, con un aumento de la sintomatología depresiva y ansiosa, o en la reducción de la autoestima (Vinaccia, Cadena, Juárez, Contreras \& Anaya, 2004).

Para concluir, dado que existe una vasta información empírica sobre el papel protector de las variables psicosociales 
positivas y de las emociones positivas para un mejor bienestar psicológico y físico en los pacientes con AR (Quiceno y Vinaccia, 2011), se hace necesario desarrollar programas de potenciación de emociones positivas, de resiliencia y de entrenamiento de técnicas de autocontrol en pacientes con esta enfermedad (Quiceno, Vinaccia y Remor, 2011; Graninger, 2015).

\section{REFERENCIAS}

Angner, E., Ray, M., Saag, K., \& Allison, J. (2009). Health and happiness among older adults. Journal of Health Psychology, 14(4), 503-512.

Angner, E., Ghandi, J., Purvis, K., Amante, D., \& Allison, J. (2013). Daily functioning, health status and happiness in older adults. Journal of Happiness Study, 14, 1563-1574.

Bautista, W., Fernandez, D., Jiménez, R., Cardozo, R., Marin, A., Soler, M. P., et al. (2015). Perfil epidemiológico de pacientes colombianos de artritis reumatoide evaluados en una clínica de especializada en atención integral. Reumatología Clínica. Doi.org/10.1016/j.reuma.2015.11.009

Bennett, P. N., Weinberg, M. K., Bridgman, T., \& Cummins, R. A. (2015). The Happiness and Subjective Well-Being of people on Haemodiaysis. Journal Renal Care, 5(1). Doi: 10.1111/jorc.12116

Broadbent, E., Petrie, K. J., Main, J., \& Weinman, J. (2006). The Brief Illness Perception Questionnaire. Journal of Psychosomatic Research, 60, 631-637.

Cruz, J. \& Torres, J. (2006). “¿De qué depende la satisfacción subjetiva de los colombianos?” Cuadernos de Economía, 45, 131-154.

CENEC (2001). El enigma de ser colombiano. Recuperado de: hhttp//: Semana.com: http://www.semana.com/wf_InfoArticulo.aspx?IdArt=93494

Covic, T., Cummings, S. R., Pallant, J. F., Manolios, N., Emery, P., Conogham, P. G., et al. (2012). Depression and anxiety in patient's whit rheumatoid arthritis: prevalence rates on a comparison of depression, anxiety and stress scale (DASS) and the hospital, anxiety and depression scale (HADS). BMC Psychiatry, 24, 12-16.

Danao, L. L., Padilla, G. V., \& Johnson, D.A. (2001). An English and Spanish quality of life measure for rheumatoid arthritis. Arthritis \& Rheumatology, 45, 167-173.

Entezami, P., Fox, D., Clapmam P., \& Chung, K. (2011). Historical Perspective on the Etiology of Rheumatoid Arthritis. Hand Clinics, 27, 1-10.

Fowler, C. \& Baas, L. S. (2006). Illness Representations in Patients with Chronic Kidney Disease on Maintenance Hemodialysis. Nephrology Nursing Journal, 33, 173-187.

Garaigordobil, M. (2015). Predictor variables of happiness and its connection with risk and protective factors for health. Frontiers in Psychology, 6, 1176. Doi: 10.3389/ fpsyg.2015.01176

Graninger, M. (2015). Behavioral training as additional therapy Approach for rheumatoid arthritis. Zeitschrift für Rheumatologie, 74(7), 579-583.

Garip, Y., Eser, F., \& Bodur, H. (2011). Health-related quality of life in rheumatoid arthritis: comparison of RAQoL with other scales in terms of disease activity, severity of pain, and functional status. Rheumatology International, 31(6), 769-772.

Gibofsky, A. (2012). Overview of epidemiology, pathophysiology, and diagnosis of rheumatoid arthritis. American Journal Management Care, 18(13), 295-302.

Goh, S. G., Rusli, B. N., \& Khalid, B. A. (2014). Diabetes quality of life perception in a multiethnic population. Quality Of Life Research, 24(7), 1677-1686.

Groarke, A., Curtis, R., Coughlan, R., \& Gsel, A. (2004). The role of perceived and actual disease status in adjustment to rheumatoid arthritis. Rheumatology, 43(9), 11421149.

Julia, A. \& Marsal, S. (2013). The genetic architecture of rheumatoid arthritis: from susceptibility to clinical subphenotype associations. Current Topics in Medicine Chemistry, 13(6), 720-731.

Hoppe, S. (2013). Chronic Illness as a source of happiness. Health, Culture and Society, 5(1). Doi 10.5195/hcs.2013.138

Isik, A., Koca, S. S., Ozturk, A., \& Mermi, O. (2007). Anxiety and depression in patients with rheumatoid arthritis. Clinical Rheumatology, 26(6), 872-878.

Lyubomirsky, S. \& Lepper, H. (1999). A measure of subjective happiness: Preliminary reliability and construct validation. Social Indicators Research, 46, 137-155.

Lyubomirsky, S., Sheldon, K. M., \& Schkade, D. (2005). Pursuing happiness: the architecture of sustainable change. General Review Psychology, 9(2), 111-131.

Machado, J. E., Ruiz, A. F., \& Medina, D. A. (2015). Epidemiología de la artritis reumatoide en una cohorte de pacientes colombianos. Revista Colombiana de Reumatología, 22(3), 148-152.

Matcham, F., Scott, I., Rayner, L., \& Hotopf, M. (2014). The Impact of rheumatoid arthritis the SF36. A Systematic review Meta-analysis. Rheumatology, 52(12), 2136-2148.

Muller, R., Terrill, A. R., Jensen, M. P., Molton, I. R., Raveslot, C., \& Ipsen, C. (2015). Happiness, pain intensity, pain interference, and distress in individuals with physical disabilities. American Journal of Physical Medicine \& Rehabilitation, 94(12), 1041-1051.

Musschenga, A. W. (1997). The relation between concepts of Quality-of-Life, Health and Happiness. The Journal of Medicine and Philosophy, 22(1), 11-28. Doi: 10.1093/ jmp/22.1.11

Nas, K., Sarak, A. J., Gur, A., Cevik, R., Altay, Z., Erdal, A., et al. (2011). Psychological status in associated Whit Health 
related quality of life in patients with rheumatoid arthritis. Journal Back Musculoskelet Rheabilitation, 24(2), 95-100.

Ozcetin, A., Ataoglu, S., Kocer, E., Yazici, S., Yaldiz, O., Ataoglul, A., et al. (2007). Effects of depression and anxiety on quality of life of patients with rheumatoid arthritis, knee osteoarthritis, fibromyalgia syndrome. Western Indian Medicine Journal, 56(2), 122-129.

Palmer, B. W., Martin, A. S., Deeep, C. A., Glorioso, D. K., \& Jeste, V. (2014). Wellness whit Wilness: Happiness in schizophrenia. Schizophrenia Research, 159(1), 151-156.

Palomera, R. (2009). Educando para la felicidad. En E.G. Fernández-Abascal (Ed.), Emociones positivas (pp. 247-274). Madrid, España: Pirámide.

Paschalides, C., Wearden, A. J., Dunkerley. R., Bundy, C., Davies, R., \& Dickens, C. M. (2004). The associations of anxiety, depression and personal illness representations with glycaemic control and health-related quality of life in patients with type 2 diabetes mellitus. Journal Psychosomatic Research, 57(6), 557-564.

Prajapati, R., Plant, D., Maskell, D., Morgan, C., Ali, F., Morgan, A., Wilson, A., et al. (2014). Impact of psychological factors on subjective disease activity assessments in patient severe whit severe rheumatoid arthritis. Arthritis Care Research, 66(6), 661-668.

Pratt, A. G. \& Isaacs, J. D. (2015). Genotyping in rheumatoid arthritis: a game changer in clinical management? Expert Review Clinical Immunology, 11(3), 303-305.

Purabdollah, M., Lakdizaji, S., Rahmani, A., Hajaliu, M., \& Anzarin, K. (2015). Relationship between sleep disorders, pain and quality of life in patients with rheumatoid arthritis. Journal of Caring Sciences, 4(3), 233-241.

Quiceno, J. M. \& Vinaccia, S. (2010). Percepción de enfermedad: Una aproximación a partir del Illness Perception Questionnaire. Revista Psicología desde el Caribe, 25, 56-83.

Quiceno, J. M. \& Vinaccia, S. (2011). Evolución de la intervención psicológica en artritis reumatoide. Revista Psicología desde el Caribe, 27(1), 160-178.

Quiceno, J. M., Vinaccia, S., Barrera, R., Latorre, R., Molina, D. C., \& Zubieta, F. A. (2013). Resiliencia, felicidad, depresión, ideación suicida y afrontamiento del estrés en estudiantes universitarios colombianos. En J. Gaxiola \& J. Palomar (Coords.), Estudios de Resiliencia en América Latina (Vols. 2) (pp. 71-88). México, D.F.: Pearson

Quiceno, J. M., Vinaccia, S., Ibáñez, J., Álvarez, A., Jiménez, J., Pinzón, L., \& Serna, A. M. (2012). Calidad de vida relacionada con la salud, resiliencia y felicidad en hombres privados de la libertad. Pensamiento Psicológico, 10(2), 23-33.

Quiceno, J. M., Vinaccia, S., \& Remor, E. (2011). Programa de potenciación de la resiliencia para pacientes con artritis reumatoide. Revista de Psicopatología y Psicología Clínica, 16(1), 27-47.
Rico, J. L., Restrepo, M., \& Molina, M. (2005). Adaptación y validación de la escala hospitalaria de ansiedad y depresión (HAD) en una muestra de pacientes con cáncer del Instituto Nacional de Cancerología de Colombia. Avances en Medición, 3(1), 73-86.

Ryan, S. (2014). Psychological effects of living with rheumatoid arthritis. Nursery Standard, 29(13), 52-59.

Ryan, S. \& McGuire, B. (2015). Psychological predictors of pain severity, pain interference, depression and anxiety in rheumatoid arthritis patients with chronic pain. British Journal of Health Psychology. Doi: 10.1111/bjhp.12171

Santiago, T., Geenen, R., Jacobs, J. W., \& Da Silva, J. A. (2015) Psychological factors associated with response to treatment in rheumatoid arthritis. Current Pharmaceutical Design, 21(2), 257-269.

Schwartzmann, L. (2003). Calidad de vida relacionada con la salud: aspectos conceptuales. Ciencia Enfermería, 2, 9-21.

Sinisterra, D. (2007). Colombian happiness: A look at life satisfaction and explanatory style. Disertación Doctoral no publicada. Florida Atlantic University, Boca Raton, FL, EE.UU.

Steptoe, A. \& Wardle, J. (2005). Positive affect and biological function in everyday life. Neurobiology Aging, 26(1), 108-112.

Steptoe, A., Deaton, A., \& Stone, A. (2015). Subjective Wellbeing, Health, and Ageing. Lancet, 385, 640-648.

Tran, P., Nilakantan, A., Foote, D., Sturgeon, D., Mackey, S., \& Johnson, K. (2015). Pain interference mediates the relationship between pain and happiness. Journal of pain, 16(4), 20. Doi: http://dx.doi.org/10.1016/j.jpain.2015.01.091

Van der Elst, K., De Cock, D., Vecoven, E., Arat, S., Meyfroidt, S., Joly, J., Moons, P., et al. (2015). Are illness perception and coping style associated with the delay between symptom onset and the first general practitioner consultation in early rheumatoid arthritis management? An exploratory study within the Care RA trial. Scandinavic Journal of Rheumatology, 23, 1-8.

Veenhoven, R. (2008). Healthy happiness. Journal of Happiness Studies, 9,449-469.

Verhagen, I., Ros, W., Steunenberg, B., \& DeWitt, N. (2014). Ethnicity does not account for differences In the health related quality of life of Turkish, Moroccan,and Molucan elderly in Netherlands. Health and Quality of Life Outcomes, 12, 138. Doi: 10.1186/s12955-014-0138-8

Vinaccia, S., Cadena, J., Juárez, F., Contreras, F., \& Anaya, J. M. (2004). Relaciones entre variables sociodemográficas, incapacidad funcional, dolor y desesperanza aprendida en pacientes con diagnóstico de artritis reumatoide. International Journal of Clinical and Health Psychology, 4(1), 91103.

Vinaccia, S., Ramírez, L., \& Toro, F. (2001). Desesperanza aprendida en pacientes con artritis reumatoide: Evolución 
y medición de un constructo. Revista Colombiana de Reumatología, 8, 443-447.

Vinaccia, S., Tobón, S., Moreno-San Pedro, E., Cadena, J., \& Anaya, J. M. (2005). Evaluación de la calidad de vida en pacientes con artritis reumatoide. International Journal of Psychology and Psychological Therapy, 5, 45-60.

Vinaccia, S., Fernández., F., Moreno, E., \& Padilla, G. (2006). Aplicación de la versión española del cuestionario Quality of Life Measure for Rheumatoid Arthritis QOL-RA en Colombia. Revista Colombiana de Reumatología, 13, 284270.

Vinaccia, S., Quiceno, J. M., \& Remor, E. (2012). Resiliencia, percepción de enfermedad, creencias y afrontamiento espiritual-religioso en relación con la calidad de vida relacionada con la salud en enfermos crónicos colombianos. Anales de Psicología, 28(2), 366-377.

Vinaccia, S., Riveros, F., Quiceno, J. M., \& Anaya, J. M. (en prensa). Análisis psicométrico de la versión española del cuestionario Quality of Life Measure for Rheumatoid Arthritis Scale (QOL-RA) en población colombiana. Enfermería clínica.

Zhao, S., Chen, Y., \& Chen, H. (2015). Sociodemographic factors associated with functional disability in outpatients with rheumatoid arthritis in Southwest China. Clinical Rheumatology, 34(5), 845-851. Doi: 10.1007/s10067-015-2896-z 